\title{
Usefulness of the Berlin, STOP, and STOP-Bang Questionnaires in the Diagnosis of Obstructive Sleep Apnea
}

\author{
Hyunju Yang, Hyunyoung Park \\ College of Nursing, Chonnam National University, Chonnam Research Institute of Nursing Science, Gwangju, Korea
}

\author{
폐쇄성수면무호흡 진단을 위한 Berlin, STOP, STOP-Bang 설문의 유용성에 관한 고찰 \\ 양현주, 박현영 \\ 전남대학교 간호대학, 간호과학연구소
}

\section{Received March 28, 2019 \\ Revised May 17, 2019 \\ Accepted May 23, 2019}

Address for correspondence Hyunyoung Park, RN, PhD College of Nursing,

Chonnam National University,

160 Baekseo-ro, Dong-gu,

Gwangju 61469, Korea

Tel: $+82-62-530-4953$

Fax: +82-62-227-4009

E-mail: hypark@jnu.ac.kr
Obstructive sleep apnea (OSA) is a chronic sleep-related breathing disorder that requires long-term management. If OSA remains untreated, it can result in serious health consequences, including increased risk of both cardiovascular and cerebrovascular diseases. Polysomnography is considered to be the gold standard for diagnosing OSA; however, it is relatively expensive, time-consuming and technically complex. Thus, there is a growing interest in the use of simple and efficient screening tools for OSA. Although screening questionnaires such as the Berlin Questionnaire, the STOP Questionnaire, and the STOP-Bang Questionnaire are widely used to assess the presence of OSA, the findings regarding their diagnostic accuracy are not consistent. This review provides a descriptive summary of the scientific studies evaluating the accuracy of diagnostic tests for OSA.

J Sleep Med 2019;16(1):11-20

\section{서 론}

폐쇄성수면무호흡(obstructive sleep apnea, OSA)은 수면 중 반복적인 상기도의 완전(무호흡) 또는 부분적(저호흡) 폐 쇄로 인해 발생하며, 수면다원검사(polysomnography, PSG) 를 통해 시간당 발생한 무호흡과 저호흡의 합이 5회 이상으 로 확인된 경우 OSA로 진단한다. ${ }^{1}$ 미국의 경우 OSA의 유병 률은 성인 남성의 $4 \%$, 성인 여성의 $2 \%$ 로 보고되었고, ${ }^{2}$ 한국 은 미국과 비슷하게 성인 남성의 $4.5 \%$, 성인 여성의 $3.2 \%$ 로 나타났다. ${ }^{3}$ OSA의 유병률은 체질량지수(body mass index, $\mathrm{BMI}$ )와 정적으로 유의한 상관관계가 있어, ${ }^{4}$ 비만 인구의 전 세계적인 증가 추세를 고려할 때 OSA 환자 수는 향후 더 늘 어날 것으로 전망된다.

$\mathrm{OSA}$ 환자는 과도한 주간 졸림증을 호소하고, OSA가 없 는 사람과 비교하였을 때 교통사고율이 3 7배까지 증가하

This is an Open Access article distributed under the terms of the Creative Commons Attribution Non-Commercial License (https://creativecommons.org/licenses/by-nc/4.0) which permits unrestricted non-commercial use, distribution, and reproduction in any medium, provided the original work is properly cited.
는 것으로 보고되었다..$^{5}$ 또한 OSA는 당뇨, 대사증후군, 부정 맥, 고혈압과 같은 만성질환의 위험인자로 알려져 있으며, 6,7 OSA가 치료되지 않을 경우 치명적인 심혈관질환이나 수면 중 돌연사가 발생할 위험이 높아질 수 있어, ${ }^{8,9} \mathrm{OSA}$ 진단 및 치료를 위한 다각적인 노력이 대두되고 있다.

OSA를 진단하는 표준 검사방법(gold standard)인 PSG는 수면 중 뇌파, 안구운동, 호흡기류, 흉부와 복부의 움직임, 심전도, 혈중산소포화농도 등을 종합적으로 측정하는 검사 로, ${ }^{1}$ 75 88\% 수준의 높은 민감도를 보인다. ${ }^{10,11}$ 그러나 절차 가 복잡하고 시간이나 인력이 많이 필요할 뿐만 아니라, 최 소 7채널 이상을 가진 장비를 갖춘 검사실 환경에서 수면기 사의 모니터링하에 수면을 취해야 하기 때문에 환자는 불편 함과 번거로움을 호소한다. 또한 건강보험이 적용되더라도 표준 PSG의 환자 부담금은 $20 \%$ 를 차지해, ${ }^{12}$ 비용에 대한 부 담도 무시할 수 없는 실정이다. 따라서 최근에는 $\mathrm{PSG}$ 를 대 체할 수 있는 검사방법이나 PSG 시행 전 OSA를 선별해낼 수 있는 검사에 대한 관심이 증가하고 있으며, OSA 의심 환 자들에 대한 선별검사를 위해 Berlin, STOP, STOP-Bang 
설문 등이 사용되고 있다. 그중 최근까지 가장 흔히 사용되 고 있는 Berlin 설문의 경우, 개발 당시의 진단적 유용성이 민감도 $86 \%$, 특이도 $77 \%$, 양성 예측도 $89 \%$, 우도비 3.79로 높게 보고되었으나, ${ }^{13}$ 추후 연구에서는 민감도와 특이도가 각각 $68 \%$ 와 $49 \%$ 로 큰 차이를 보였다. ${ }^{14}$

$\mathrm{OSA}$ 의 진단과 치료에 건강보험이 적용됨에 따라 $\mathrm{OSA}$ 의 심 환자의 수면클리닉 이용이 점차 증가할 것으로 예측되는 상황에서 가장 신뢰할 만한 선별도구를 사용하여 OSA 진단 에 소요되는 비용과 인력, 환자의 불편감이나 부담을 감소시 키려는 노력은 의미가 있을 것이다. 따라서 본 종설은 최근 10년간의 연구를 통해 Berlin, STOP, STOP-Bang 설문의 $\mathrm{OSA}$ 에 대한 진단적 유용성을 연구 대상에 따라 지역사회 인구와 수면클리닉 내원 환자로 구분하여 확인하고, 설문 간 유용성의 차이를 비교 고찰하고자 한다.

\section{본 론}

Berlin, STOP, STOP-Bang 설문은 OSA 선별에 일반적 으로 사용되는 도구로, ${ }^{15}$ 세 가지 설문의 문항 구성은 Table 1 과 같다. 먼저 Berlin 설문은 한국을 비롯한 여러 나라에서 $\mathrm{OSA}$ 진단 시 가장 흔히 사용되는 선별도구 중 하나로, ${ }^{16}$ 코 골이와 무호흡, 주간 졸림증과 피로도, 고혈압과 비만도 같 은 수면무호흡 관련 증상에 대한 질문이 총 3개의 카테고리 로 구성되어 있다. ${ }^{13}$ Category 1 은 코골이 유무 및 정도와 빈 도, 목격된 무호흡 등에 관한 5 개 질문으로 구성되어 있고, 이 중 최소 2 가지 질문에 양성으로 응답할 경우 category 1 을
양성으로 판정한다. Category 2는 수면 후 피로도, 각성 시 피로도, 졸음운전의 유무와 빈도에 관한 4개 질문으로 구성 되어 있으며, 역시 최소 2가지 질문에 양성이면 category 2 를 양성으로 판정한다. Category 3 은 고혈압과 BMI에 관한 질문으로 구성되어 있으며, 고혈압이 존재하거나 BMI가 30 $\mathrm{kg} / \mathrm{m}^{2}$ 를 초과할 경우 양성으로 판정한다. 그리고 카테고리 별 결과를 바탕으로 총 3 개 카테고리 중 2 개 이상에서 양성 일 경우 '고위험군'으로, 2 개 미만일 경우에는 '저위험군'으 로 판정한다. ${ }^{13}$

STOP 설문은 코골이(snoring), 주간 피로도(tiredness), 목 격된 무호흡(observed apnea), 고혈압(blood pressure) 여부 에 관한 총 4가지 질문에 '예', '아니오'로 답하게 되어 있으 며, 이 중 2개 이상의 질문에서 ‘예’로 응답한 경우에는 ‘고위 험군'으로, 1 개 이하의 경우에는 '저위험군'으로 판정한다. 반면 STOP-Bang 설문은 STOP 설문에 4개 문항 즉, BMI (BMI $\left.>35 \mathrm{~kg} / \mathrm{m}^{2}\right)$, 연령 (age>50세), 목둘레(neck circumference $>41 \mathrm{~cm}$ ), 성별(gender, male)을 추가한 총 8 가지 질 문으로 구성되어 있으며, 그중 3개 이상에서 '예'로 응답한 경 우 '고위험군'으로, 3 개 미만의 경우에는 '저위험군'으로 판 정한다. ${ }^{17}$

\section{지역사회 인구에서 Berlin, STOP, STOP-Bang 설문의 진단적 유용성}

본 종설에서는 지역사회 인구 기반 연구(population-based study)로 프랑스, 한국, 싱가포르, 미국에서 시행된 5개 연구 를 고찰하였다(Table 2). ${ }^{18-22}$ 임상에서 수면연구를 시행할 때

Table 1. Summary of obstructive sleep apnea questionnaires

\begin{tabular}{lll}
\hline Berlin questionnaire & STOP questionnaire & STOP-Bang questionnaire \\
\hline C1. Presence of snoring & S. Presence of snoring & S. Presence of snoring \\
$\begin{array}{l}\text { C1. Severity of snoring } \\
\text { C1. Frequency of snoring }\end{array}$ & \\
$\begin{array}{l}\text { C1. Snoring bothersome to others } \\
\text { C1. Observed apneas or choking }\end{array}$ & O. Observed apneas or choking & \\
$\begin{array}{l}\text { C2. Sleepiness or fatigue after sleep } \\
\text { C2. Wake-time sleepiness or fatigue }\end{array}$ & T. Tiredness or sleepiness & O. Observed apneas or choking \\
C2. Presence of drowsy driving & & T. Tiredness or sleepiness \\
C2. Frequency of drowsy driving & & \\
C3. Hypertension & P. Hypertension & \\
C3. Obesity (BMI $\left.>30 \mathrm{~kg} / \mathrm{m}^{2}\right)$ & & P. Hypertension \\
& & B. Obesity (BMI $\left.>35 \mathrm{~kg} / \mathrm{m}^{2}\right)$ \\
& & A. Age $>50 \mathrm{yr}$ \\
\hline
\end{tabular}

C1: category 1, C2: category 2, C3: category 3, BMI: body mass index 
Yang $\mathrm{H}$ et al.

Table 2. Diagnostic accuracy of screening tools in the general population

\begin{tabular}{|c|c|c|c|c|c|c|c|}
\hline \multirow{2}{*}{\multicolumn{2}{|c|}{ Screening tool }} & \multirow{2}{*}{$\begin{array}{c}\text { Sforza et al., } 2011^{18} \\
\text { Berlin }\end{array}$} & \multirow{2}{*}{$\frac{\text { Kang et al., } 2013^{19}}{\text { Berlin }}$} & \multirow{2}{*}{$\begin{array}{c}\text { Tan et al., } 2017^{20} \\
\text { Berlin }\end{array}$} & \multicolumn{2}{|c|}{ Silva et al., $2011^{* 22}$} & \multirow{2}{*}{$\begin{array}{c}\text { Tan et al., 2016 } 21 \\
\text { STOP-Bang }\end{array}$} \\
\hline & & & & & STOP & STOP-Bang & \\
\hline \multicolumn{2}{|c|}{ Number } & 643 & 1,305 & 242 & 4,770 & 4,770 & 242 \\
\hline \multicolumn{2}{|c|}{ Research site } & France & Korea & Singapore & USA & USA & Singapore \\
\hline \multicolumn{2}{|c|}{ Age (yr) } & $65.6 \pm 0.1$ & $52.8 \pm 16.6$ & $48.3 \pm 14.0$ & $62.4 \pm 10.3$ & $62.4 \pm 10.3$ & $48.3 \pm 14.0$ \\
\hline \multicolumn{2}{|c|}{ Male (\%) } & 40.9 & 47.7 & 50.4 & 51.5 & 51.5 & 50.4 \\
\hline \multicolumn{2}{|c|}{ BMI $\left(\mathrm{kg} / \mathrm{m}^{2}\right)$} & $25.3 \pm 0.2$ & $22.8 \pm 4.9$ & $26.2 \pm 5.0$ & - & - & $26.2 \pm 5.0$ \\
\hline \multicolumn{2}{|c|}{ Level of sleep study } & Level III & Level I & Level III & Level II & Level II & Level III \\
\hline \multicolumn{2}{|c|}{$\begin{array}{l}\text { Hypopnea defined } \\
\text { at OD }(\%)\end{array}$} & $\geq 3$ & $\geq 3$ & $\geq 3$ & $\geq 4$ & $\geq 4$ & $\geq 3$ \\
\hline \multicolumn{8}{|c|}{ AHI threshold (events/hr) } \\
\hline \multirow[t]{4}{*}{$\geq 5$} & SN/SP (\%) & $-/-$ & $69.0 / 83.0$ & $-1-$ & $-1-$ & $-/-$ & $-1-$ \\
\hline & PPV/NPV (\%) & $-1-$ & $-1-$ & $-1-$ & $-1-$ & $-/-$ & $-1-$ \\
\hline & $\mathrm{LR}(+) / \operatorname{LR}(-)$ & $-/-$ & $4.1^{\dagger} / 0.4^{\ddagger}$ & $-1-$ & $-1-$ & $-/-$ & $-1-$ \\
\hline & AUC & - & - & - & $-1-$ & $-/-$ & $-1-$ \\
\hline \multirow[t]{4}{*}{$\geq 15$} & SN/SP (\%) & $76.7 / 39.3$ & $89.0 / 63.0$ & $58.8 / 77.6$ & $62.0 / 56.3$ & $87.0 / 43.3$ & $66.2 / 74.7$ \\
\hline & PPV/NPV (\%) & $63.2 / 55.4$ & $-1-$ & $50.6 / 82.9$ & $-1-$ & $-1-$ & $50.6 / 85.0$ \\
\hline & $\mathrm{LR}(+) / \mathrm{LR}(-)$ & $1.3^{\dagger} / 0.6^{\ddagger}$ & $2.4^{\dagger} / 0.2^{\ddagger}$ & $2.6 / 0.5$ & $1.4 / 0.7$ & $1.5 / 0.3$ & $2.6 / 0.5$ \\
\hline & AUC & 0.60 & - & 0.68 & 0.58 & 0.64 & 0.70 \\
\hline \multirow[t]{4}{*}{$\geq 30$} & SN/SP (\%) & $-1-$ & $-1-$ & $76.9 / 72.7$ & $68.8 / 59.5$ & $70.4 / 59.5$ & $69.2 / 67.1$ \\
\hline & PPV/NPV (\%) & $-/-$ & $-1-$ & $25.3 / 96.3$ & $-1-$ & $-1-$ & $20.2 / 94.8$ \\
\hline & $\mathrm{LR}(+) / \mathrm{LR}(-)$ & $-1-$ & $-1-$ & $2.8 / 0.3$ & $1.5 / 0.7$ & $1.7 / 0.5$ & $2.1 / 0.5$ \\
\hline & AUC & - & - & 0.75 & 0.65 & 0.66 & 0.68 \\
\hline
\end{tabular}

${ }^{*}$ measured by Respiratory Disturbance Index, the likelihood ratio for a positive result=SN/(1-SP), tthe likelihood ratio for a negative result=(1-SN)/SP. BMI: body mass index, OD: oxygen desaturation, AHI: Apnea-Hypopnea Index, SN: sensitivity, SP: specificity, PPV: positive predictive value, NPV: negative predictive value, LR(+): positive likelihood ratio, LR(-): negative likelihood ratio, AUC: area under the receiver operating characteristic curve

제한점 중 하나는 연구 대상자에서 남성의 비중이 크다는 점이다. ${ }^{3,23,24}$ 그러나 본 종설의 인구 기반 연구들은 남성의 비율이 40.9 51.5\%로 남녀 간 성비가 비교적 비슷하기 때문 에 성비 불균형에 따른 결과 편향은 최소화되었을 것으로 사료된다.

우선 Berlin 설문을 사용한 세 연구에서 무호흡-저호흡 지수(Apnea-Hypopnea Index, AHI) 15의 절단 값(cut-off value)을 기준으로 한 설문의 진단적 유용성은 민감도는 $58.8 \sim 89.0 \%$, 특이도는 39.3 77.6\%로 연구 간 큰 차이가 있 었다. ${ }^{18-20}$ 평균 65.6세의 노인 인구를 대상으로 한 Sforza 등 의 연구에서 연구 대상 전체에 대한 민감도, 특이도, 양성예 측도 및 음성예측도는 각각 $77.6 \%, 39.3 \%, 63.2 \%, 55.4 \%$ 였 고, 성별에 따라 남성은 $86.9 \%, 27.9 \%, 71.2 \%, 51.1 \%$ 를, 여성 은 $67.4 \%, 44.6 \%, 55.8 \%, 56.9 \%$ 를 나타내, ${ }^{18}$ Berlin 설문은 남 성 노인에 비해 여성 노인에서 낮은 민감도와 양성예측도를 나타냄을 알 수 있었다. 이는 OSA 환자의 약 $50 \%$ 가 불면증 과 관련된 증상(insomnia-like symptoms)을 호소하는데, 불
면증 증상은 OSA 증상과 유사해 불면증 유병률이 높은 여 성 OSA 환자가 수면에 들기 힘들거나, 수면 시 자주 깨거나, 수면을 지속하기 힘든 것과 같은 불면증의 증상들을 수면무 호흡 증상으로 잘못 판단하여 선별도구의 유용성에 영향을 미쳤을 가능성이 있다. ${ }^{23,24}$ 따라서 여성 노인을 대상으로 Berlin 설문을 사용할 때는 환자가 불면증이나 다른 수면 관 련 질환에 기왕력이 있는지에 대한 사전조사가 필요하다.

Kang 등의 연구에서 AHI 절단 값을 5 로 하여 Berlin 설문 의 진단적 유용성을 평가하였을 때 민감도는 $69.0 \%$, 특이도 는 $83.0 \%$ 였고, 절단 값을 10으로 하였을 때 민감도는 $79.0 \%$, 특이도는 $67.0 \%$ 였으며, 절단 값을 15 로 하였을 때 민감도는 $89.0 \%$, 특이도는 $63.0 \%$ 로 나타나, OSA 진단 시 $\mathrm{AHI}$ 절단 값을 엄격히 적용할수록 Berlin 설문의 민감도는 높아지나 특이도는 낮아지는 것으로 나타났다. ${ }^{19}$ 국민건강보험에서 양 압기의 급여 적용 기준은 성인의 경우 제1형 PSG(Level I) 결과에서 $\mathrm{AHI}$ 가 15 이상이거나, 또는 5 이상이면서 수면무 호흡 관련 증상(불면증, 주간 졸음, 인지기능 감소, 기분장 
애, 고혈압, 빈혈성 심장질환, 뇌졸중 기왕력, 산소포화도 $85 \%$ 미만)가 하나 이상인 경우에 해당한다. ${ }^{25}$ 따라서 OSA 관련 증상을 동반한 환자가 Berlin 설문에서 OSA '저위험군' 으로 분류되었다면 그 결과는 위음성(false negative)일 가능 성도 있으므로, 이로 인해 환자가 양압기 적용 기회를 놓치 지 않도록 선별검사의 결과를 신중히 검토해야 할 것이다.

Sforza 등과 Tan 등의 연구에서는 검사자에 의한 감시 없 이(Level III: In-home portable sleep monitoring) 자택용 수면무호흡 검사기(Home Sleep Apnea Test, HSAT)를 통 해 수면무호흡을 측정한 반면, $18,20 \mathrm{Kang}$ 등의 연구는 수면검 사실에서 최소 7채널 이상을 가진 PSG 장비(Level I: Standard in-lab PSG)로 OSA를 진단하였다. ${ }^{19}$ 선행연구에 따르 면, HSAT는 표준 PSG에 비해 저호흡의 수준을 저평가하여 $17 \%$ 정도의 위음성(false negative)을 보이고, 산소포화도 저 하 원인이 수면무호흡인지도 확신할 수 없다고 하였다. ${ }^{26}$ 그 러므로 두 연구에서 보고된 음성예측도 $(55.4 \%, 82.9 \%)$ 는 실 제보다 높게 측정되었을 가능성이 있다.

Tan 등은 지역사회 인구를 대상으로 Berlin 설문과 STOPBang 설문을 각각 사용하였는데, AHI 절단 값을 15 로 하였 을 때 민감도, 특이도, 양성/음성 예측도, 양성/음성 우도비, area under the receiver operating characteristic(ROC) curve (AUC)는 두 설문이 비슷한 결과를 나타냈고, $\mathrm{AHI}$ 절단 값 을 30으로 적용하였을 때 민감도, 특이도, 양성예측도, 양성 우도비, AUC는 Berlin 설문 $(76.9 \%, 72.7 \%, 25.3 \%, 2.8,0.75)$ 이 STOP-Bang 설문(69.2\%, 67.1\%, 20.2\%, 2.1, 0.68)에 비해 더 높게 나타났다. ${ }^{20,21}$ 그러나 약 5,000명의 미국인을 대상으 로 STOP-Bang 설문에 AHI 절단 값 30을 적용한 Silva 등 의 연구에서는 민감도, 특이도, 양성/음성 예측도, $\mathrm{AUC}$ 가 Tan 등의 연구와 비슷한 결과를 나타내고 있어, STOPBang 설문은 지역사회 인구 대상의 중증 OSA 진단 시 인종 이나 지역적 차이의 영향을 받지 않는 안정적인 도구라 판 단된다. ${ }^{21,22}$

지역사회 인구를 대상으로 Berlin, STOP, STOP-Bang 설 문을 사용하였을 때, STOP 설문이 AHI 절단 값 15 와 30 의 기준 모두에서 진단적 유용성이 가장 낮은 것으로 나타나, ${ }^{18-22}$ 향후 지역사회 인구 기반 연구에는 STOP 설문보다 정확도 가 높은 Berlin이나 STOP-Bang 설문을 사용하는 것이 더 바람직해 보인다. 하지만 아직까지 지역사회 인구 기반 연구 에서 STOP과 STOP-Bang 설문의 사용 빈도는 Berlin 설문 에 비해 낮고, 국내에서도 STOP이나 STOP-Bang 설문을 이용하여 OSA 진단적 유용성을 파악한 연구는 미비하였으 므로, 향후 국내 지역사회 인구를 대상으로 OSA 감별을 위 한 Berlin, STOP, STOP-Bang 설문 간 진단적 유용성의 차
이를 확인하는 연구가 더욱 필요할 것으로 사료된다.

요약하자면 지역사회 인구를 대상으로 OSA 선별검사를 시행할 때 Berlin 설문의 진단적 유용성은 연령이나 성별, 수면 관련 질환의 기왕력, $\mathrm{AHI}$ 의 절단 값 수준, 진단검사 장 비의 유형에 따라 달라질 수 있음이 고려되어야 한다. 한편 중증 OSA 감별에는 Berlin과 STOP-Bang 설문이 유용한 도구로 보고되고는 있으나, 국내 지역사회 인구를 대상으로 한 근거가 아직 미비하므로 STOP과 STOP-Bang 설문의 진 단적 유용성에 대한 추후 연구가 보다 활발히 이루어져야 할 것이다.

\section{수면클리닉 내원 환자에서 Berlin, STOP, STOP-Bang 설문의 진단적 유용성}

\section{Berlin 설문의 진단적 유용성}

본 종설에 포함된 수면클리닉 내원 환자(sleep clinic population) 대상 연구 중 Berlin 설문을 적용한 연구는 13편이 있었고, 이들 연구는 여러 국가에서 다양한 연구방법과 기준 점을 가지고 OSA 진단을 위한 설문의 유용성을 평가하였다 (Table 3). ${ }^{327-38}$ 우리나라와 홍콩을 포함한 동아시아 지역의 연구에서는 연구 대상자의 평균 연령, 성별, 평균 BMI가 비 슷하게 나타났으며, 환자의 대부분은 중년 남성(73.6 84.9\%) 이었고, 평균 $\mathrm{BMI}$ 는 $24.7 \pm 3.5 \sim 26.5 \pm 4.1 \mathrm{~kg} / \mathrm{m}^{2}$ 로 정상에서 과체중 사이에 있었다. 3,27,29,33 반면, 유럽이나 북미 등에서 수 행된 연구에서는 연구 대상자의 평균 BMI가 31.0 \pm 6.6 $37.8 \pm 9.5 \mathrm{~kg} / \mathrm{m}^{2}$ 로 경도 비만에서 중증도 비만에 해당하였 다. 선행연구에 따르면 $\mathrm{BMI} \geq 30 \mathrm{~kg} / \mathrm{m}^{2}$ 인 비만 환자의 $50 \%$ 이상이 OSA를 가지고 있으며, 체중이 $10 \%$ 증가하면 중등도 $\mathrm{OSA}(\mathrm{AHI} \geq 15)$ 의 발병 위험이 6 배가량 높았다. ${ }^{39,40}$ 따라서 상대적으로 덜 비만한 동아시아의 연구 대상자는 Berlin 설 문의 category 3 에서 양성 판정을 받을 확률이 낮으므로, 비 만율이 높은 서양 환자를 대상으로 한 Berlin 설문의 진단적 유용성 결과는 한국인 대상의 연구결과와 다를 수 있음을 고려해야 한다. 만약 수면클리닉 내원 환자가 OSA와 관련 된 임상 증상이 뚜렷하게 나타나지만 비만도가 $30 \mathrm{~kg} / \mathrm{m}^{2}$ 미 만이면서 Berlin 설문에서 음성을 나타냈다면, STOP이나 STOP-Bang 설문과 같은 다른 선별도구를 활용한 추가 검 사를 시행해보아야 할 것이다.

다음은 산소포화도 감소 정도에 따라 저호흡을 진단하는 American Academy of Sleep Medicine(AASM)의 저호흡 진단기준을 통해 Berlin 설문의 진단적 유용성을 살펴보고 자 한다. AASM에 따르면 저호흡은 뇌파상 각성이 있거나 $3 \%$ 이상의 산소포화도 감소가 관찰된 경우는 권고 수준(rec- 
Table 3. Diagnostic accuracy of the Berlin questionnaire in a sleep clinical setting

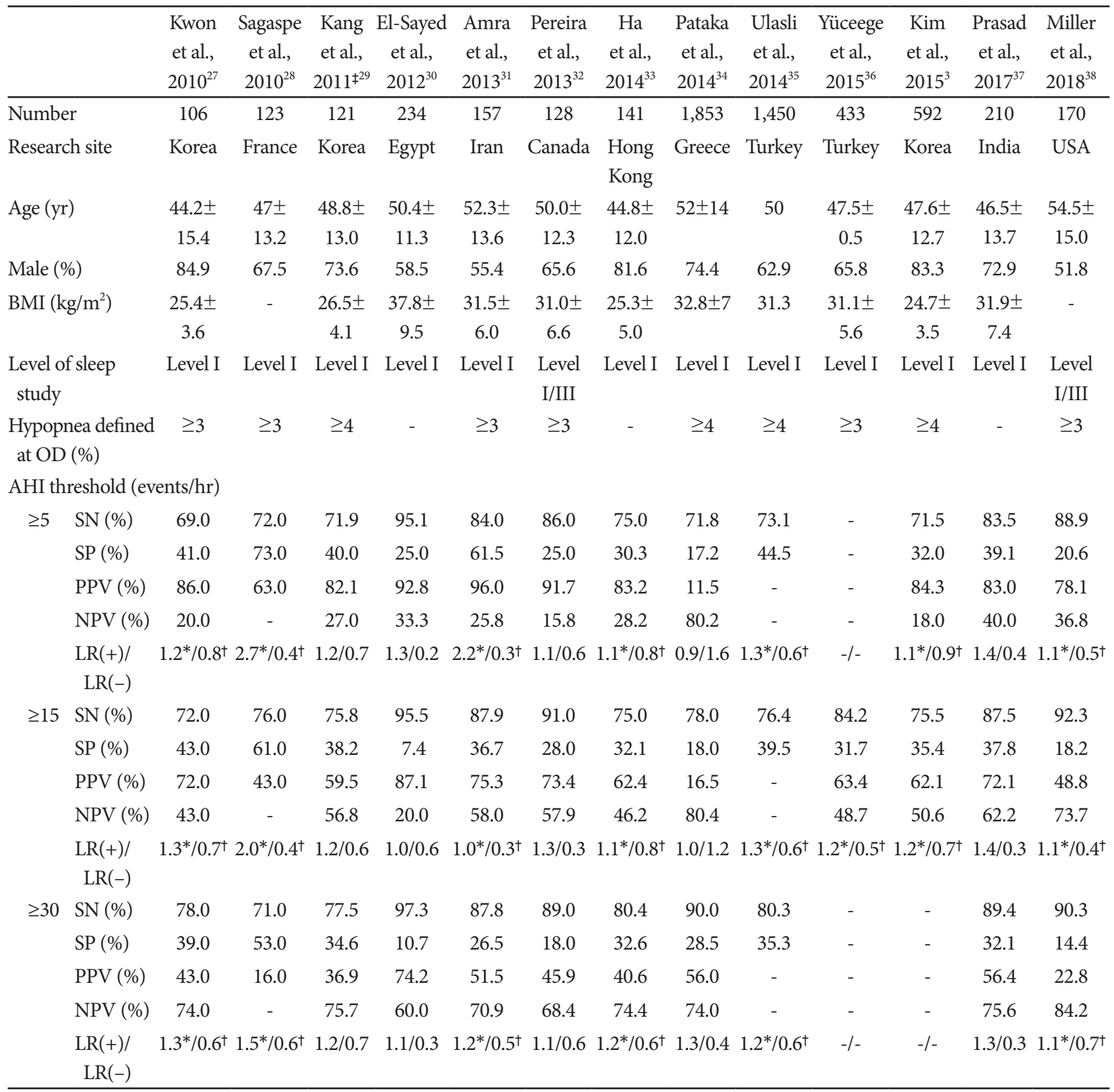

${ }^{*}$ the likelihood ratio for a positive result=SN/(1-SP), ${ }^{t}$ the likelihood ratio for a negative result=(1-SN)/SP, ${ }^{\ddagger}$ area under the receiver operating characteristic curve: AHI 5, 0.56; AHI 15, 0.57; AHI 30, 0.56. BMI: body mass index, OD: oxygen desaturation, AHI: Apnea-Hypopnea Index, SN: sensitivity, SP: specificity, PPV: positive predictive value, NPV: negative predictive value, LR(+): positive likelihood ratio, LR(-): negative likelihood ratio

ommended), $4 \%$ 이상 산소포화도가 감소되는 경우는 받아 들일 만한 수준(acceptable)에 해당한다. ${ }^{41}$ 한국에서 수행된 연구 중 Kang 등과 Kim 등의 연구는 $\mathrm{AASM}$ 의 저호흡 진단 기준으로 $4 \%$ 이상의 산소포화도 감소를, ${ }^{3,2} \mathrm{Kwon}$ 등의 연 구는 $3 \%$ 이상의 산소포화도 감소를 적용하여 진단적 유용 성을 보고하였다. ${ }^{27}$ 세 연구에서 민감도와 특이도는 비슷하 게 나타났으나, 양성예측도는 $3 \%$ 이상의 산소포화도 감소를 적용한 연구가 $4 \%$ 이상의 산소포화도 감소를 적용한 연구에
비해 더 높았다. 다른 국가에서 수행된 연구들에서는 Berlin 설문의 진단적 유용성이 저호흡 진단기준을 $4 \%$ 이상의 산 소포화도 감소로 적용하였을 때보다, ${ }^{34,35} 3 \%$ 이상 산소포화 도 감소로 적용하였을 때 더 높게 나타났다. ${ }^{28,31,32,36,38} \mathrm{AHI}$ 절 단 값을 5 로 하였을 때 $4 \%$ 이상의 산소포화도 감소를 적용 한 연구에서 양성예측도는 $11.5 \sim 84.3 \%$ 로 보고되어, $32,28,34$ 연 구 간 Berlin 설문의 타당도 차이는 큰 것으로 나타났다. 이 를 종합해 보면 Berlin 설문의 진단적 유용성은 AASM 저호 
흡 진단기준에서 $4 \%$ 이상보다 $3 \%$ 이상의 산소포화도 감소 를 적용했을 때 더 높은 것으로 사료된다.

동아시아에서 수행된 4개 연구에서 Berlin 설문의 민감도, 특이도, 양성예측도 및 음성예측도는 모두 유사하게 나타났 으며, $\mathrm{AHI}$ 절단 값을 $5,15,30$ 으로 달리하여도 민감도(69.0 80.4\%)와 특이도(30.3 43.0\%)의 차이는 크지 않았다. 3,27,29,33 하지만 질병의 유병률에 영향을 받지 않고 선별도구의 정확 도를 평가할 수 있는 장점을 가진 양성우도비와 음성우도비 를 고려한다면, Berlin 설문이 OSA를 선별하는 수준은 그리 높지 않았다. 본 종설에 포함된 13 개의 연구 중 5 개 연구에 서만 우도비를 보고하고 있어, $29,30,32,34,37$ 본 연구자들이 민감 도와 특이도를 이용해 우도비를 직접 산출하여 비교해 본 결과, Berlin 설문의 우도비는 두 연구에서만 의미 있는 결 과를 보였고 Sagaspe 등과 Amra 등의 연구에서 양성우도비 는 2.2 2.7, 음성우도비는 0.3 0.4로 선별도구의 정확도가 '약함' 수준에 머물렀다. ${ }^{28,31}$ 또한 Kang 등의 연구에서 $\mathrm{AHI}$ 절단 값을 $5,15,30$ 으로 달리 하였을 때 AUC는 각각 0.56 , $0.57,0.56$ 으로 약 0.5 수준에 머물러 한국인의 OSA 진단을 위한 Berlin 설문의 유용성은 높지 않았다. ${ }^{29}$ 이는 Berlin 설 문이 OSA를 유발하는 해부학적, 생리학적, 인구학적 요인 을 고려하지 않고, 수면무호흡에 관한 비특이적 증상을 질문 에 포함하고 있어 나타난 결과로 생각된다. OSA의 위험요 인은 노인, 목둘레가 큰 경우, 비만도가 높은 경우 등이며, ${ }^{42}$ $\mathrm{OSA}$ 를 가진 환자는 수면 중 반복된 저산소증과 그로 인해 발생한 수면각성으로 과도한 주간 졸음이나 피로를 호소하 는 특징적 증상을 나타낸다. Berlin 설문은 비만도 유무를 category 3에 포함시켰으나, 그 외 성별, 연령, 목둘레 등과 같은 위험요인은 평가 항목으로 고려하지 않는다. 또한 category 2에서는 ‘수면 후 졸음/피로도', '각성 시 졸음/피로도', '졸음 운전'과 같은 낮 동안의 졸림증에 대해 묻고 있으나, 이는 수면무호흡뿐만 아니라 다른 질환에서도 발생 가능한 일반적 증상으로 수면무호흡과 관련한 특이적 증상이라 보 기는 힘들다. 특히, 졸음운전에 대해 묻는 질문은 사회적 규 범에 용납되지 않는 행위를 감추기 위해 거짓으로 대답하는 측정도구의 체계적 오차(systematic error)를 발생시킬 가능 성이 있다. ${ }^{43} \mathrm{Amra}$ 등의 연구에 따르면 Berlin 설문의 내적 일관성을 살펴보았을 때 도구의 질문 간 상관성은 0.2 로 낮 았으나, ‘졸음 운전'의 항목을 배제할 경우에는 질문 간 상관 성이 0.6 으로 상승하는 것으로 나타났다. ${ }^{31}$ 한국인 대상의 연 구에서도 비슷한 결과를 보고하였는데, Category 1과 3은 $\mathrm{AHI}$ 와 통계적으로 유의한 상관성을 보인 반면, 수면 후 피로 도, 각성 시 피로도, 졸음운전의 유무와 빈도로 구성된 category 2 와 $\mathrm{AHI}$ 의 상관성은 통계적으로 유의하지 않았다. ${ }^{29}$
따라서 추후 연구에서는 Berlin 설문을 구성하는 각 문항과 $\mathrm{AHI}$ 와의 상관성을 살핀 후 상관계수가 낮은 질문은 배제하 여 Berlin 설문의 타당성을 향상시킨 다음 진단적 유용성을 다시 살펴볼 필요가 있을 것으로 사료된다.

\section{AHI 기준에 따른 Berlin, STOP, STOP-Bang 설문의 진단적 유용성 비교}

본 종설에서 AHI 절단 값 $5,15,30$ 을 기준으로 Berlin, STOP, STOP-Bang 설문의 진단적 유용성을 비교 고찰한 결과는 다음과 같다(Table 4-6).

민감도는 AHI 절단 값 5 를 기준으로 하였을 때 Berlin 설 문이 69.0 95.1\%, STOP 설문이 74.8 97.0\%, STOP-Bang 설문이 81.1 97.6\%로, 세 가지 설문 중 Berlin 설문이 가장 낮은 민감도를 나타냈고(Table 4), AHI 절단 값을 15(Table 5)와 30(Table 6)으로 적용하였을 때도 동일하게 Berlin 설 문이 가장 낮은 민감도를 보였다. $3,27,30,32-34,37,38$ 설문의 진단적 유용성을 비교한 연구의 대부분에서 STOP-Bang 설문이 $\mathrm{OSA}$ 선별에 가장 정확한 도구로, Berlin 설문은 민감도가 가장 낮은 도구로 보고되었다. $32-34,37,38$ 한국인을 대상으로 한 연구에서는 $\mathrm{AHI}$ 절단 값에 따른 Berlin 설문의 민감도가 69.0 78.0\%로 나타나, STOP 설문의 97.0 98.0\%나 STOPBang 설문의 97.0 98.0\%보다 낮게 측정되는 결과를 보였으 며, 특이도는 Berlin 설문이 32.0 43.0\%로 STOP 설문의 12.0 35.0\%나 STOP-Bang 설문의 10.6 18.6\%보다 높게 나 타났다. 327 이는 STOP이나 STOP-Bang 설문 항목 중 '피곤 함, '고혈압', ‘50세 이상의 연령', '남성'의 여부가 OSA의 위 험요인이지만 질병 특이성이 떨어져, OSA의 위험이 높지 않은 환자를 배제시키지 못했기 때문일 것으로 판단된다. STOP과 STOP-Bang 설문의 우도비를 살펴보면 양성우도 비는 모두 2.0 미만으로 나타났고, 음성우도비는 대부분의 연구에서 0.5 이상의 수치를 나타냈다. 3,27,30,32-34,37,38 즉, STOP 설문과 STOP-Bang 설문을 통해 OSA로 진단된 경우 실제 질환이 있을 진단 정확도(rule-in)와 OSA가 없다고 진단된 경우 실제로 질환이 없을 진단 정확도(rule-out)는 떨어진다 고 할 수 있다.

선별검사의 진단적 유용성은 ROC curve의 아래 면적 (AUC)을 측정한 값으로도 판단할 수 있으며, AUC 수치가 0.5 일 경우 ‘비정보적', $0.5<\mathrm{AUC} \leq 0.7$ 일 경우 '덜 정확한', $0.7<\mathrm{AUC} \leq 0.9$ 일 경우 ‘중등도의 정확한', $0.9<\mathrm{AUC}<1$ 일 경우 '매우 정확한', 1 일 경우 ‘완벽한' 검사로 분류한다.44 Berlin, STOP, STOP-Bang 설문의 AUC 수치는 AHI 절단 값 5 를 적용하였을 때 $0.45,0.49,0.48$ 로 모두 0.5 이하의 값 을 나타냈고, 절단 값 15 를 적용한 경우에도 각각 $0.48,0.50$, 
Table 4. Diagnostic accuracy of screening tools in a sleep clinical setting (Apnea-Hypopnea Index $\geq 5$ events/hr)

\begin{tabular}{|c|c|c|c|c|c|c|c|c|}
\hline & $\begin{array}{c}\text { Kwon et al., } \\
2010^{27}\end{array}$ & $\begin{array}{c}\text { El-Sayed } \\
\text { et al., } 2012^{30}\end{array}$ & $\begin{array}{c}\text { Pereira et al., } \\
2013^{32}\end{array}$ & $\begin{array}{c}\text { Ha et al., } \\
2014^{33}\end{array}$ & $\begin{array}{c}\text { Pataka et al., } \\
2014^{\ddagger^{34}}\end{array}$ & $\begin{array}{l}\text { Kim et al., } \\
2015^{3}\end{array}$ & $\begin{array}{c}\text { Prasad et al., } \\
2017^{37}\end{array}$ & $\begin{array}{l}\text { Miller et al., } \\
2018^{38}\end{array}$ \\
\hline \multicolumn{9}{|l|}{ Berlin } \\
\hline SN (\%) & 69.0 & 95.1 & 86.0 & 75.0 & 71.8 & 71.5 & 83.5 & 88.9 \\
\hline SP (\%) & 41.0 & 25.0 & 25.0 & 30.3 & 17.2 & 32.0 & 39.1 & 20.6 \\
\hline PPV (\%) & 86.0 & 92.8 & 91.7 & 83.2 & 11.5 & 84.3 & 83.0 & 78.1 \\
\hline NPV (\%) & 20.0 & 33.3 & 15.8 & 28.2 & 80.2 & 18.0 & 40.0 & 36.8 \\
\hline $\operatorname{LR}(+) / \operatorname{LR}(-)$ & $1.2^{*} / 0.8^{\dagger}$ & $1.3 / 0.2$ & $1.1 / 0.6$ & $1.1^{*} / 0.8^{\dagger}$ & $0.9 / 1.6$ & $1.1^{*} / 0.9^{\dagger}$ & $1.4 / 0.4$ & $1.1^{*} / 0.5^{\dagger}$ \\
\hline \multicolumn{9}{|l|}{ STOP } \\
\hline SN (\%) & 97.0 & 91.7 & - & 74.8 & 91.7 & - & 87.8 & - \\
\hline SP (\%) & 35.0 & 25.0 & - & 50.0 & 6.4 & - & 43.5 & - \\
\hline PPV (\%) & 89.0 & 92.6 & - & 85.6 & 12.8 & - & 84.7 & - \\
\hline NPV (\%) & 67.0 & 22.7 & - & 33.3 & 84.0 & - & 50.0 & - \\
\hline $\operatorname{LR}(+) / \operatorname{LR}(-)$ & $1.5^{*} / 0.1^{\dagger}$ & $1.2 / 0.3$ & - & $1.5^{*} / 0.5^{\dagger}$ & $1.0 / 1.3$ & - & $1.6 / 0.3$ & - \\
\hline \multicolumn{9}{|l|}{ STOP-Bang } \\
\hline $\mathrm{SN}(\%)$ & - & 97.6 & 90.0 & 81.1 & 90.0 & 97.0 & 89.0 & 94.4 \\
\hline SP (\%) & - & 26.3 & 42.0 & 57.1 & 4.9 & 18.6 & 43.5 & 20.6 \\
\hline PPV (\%) & - & 93.4 & 93.7 & 88.2 & 12.2 & 85.9 & 84.9 & 79.1 \\
\hline NPV (\%) & - & 50.0 & 29.4 & 43.2 & 76.8 & 54.6 & 52.6 & 53.9 \\
\hline $\operatorname{LR}(+) / \operatorname{LR}(-)$ & - & $1.3 / 0.1$ & $1.5 / 0.2$ & $1.9^{*} / 0.3^{\dagger}$ & $0.9 / 2.0$ & $1.2^{*} / 0.2^{\dagger}$ & $1.6 / 0.3$ & $1.2^{*} / 0.3^{\dagger}$ \\
\hline
\end{tabular}

*the likelihood ratio for a positive result=SN/(1-SP), ${ }^{t}$ the likelihood ratio for a negative result= $(1-\mathrm{SN}) / \mathrm{SP}$, ${ }^{\ddagger}$ area under the receiver operating characteristic curve: Berlin, 0.45; STOP, 0.49; STOP-Bang, 0.48. SN: sensitivity, SP: specificity, PPV: positive predictive value, NPV: negative predictive value, $\mathrm{LR}(+)$ : positive likelihood ratio, $\operatorname{LR}(-)$ : negative likelihood ratio

Table 5. Diagnostic accuracy of screening tools in a sleep clinical setting (Apnea-Hypopnea Index $\geq 15$ events/hr)

\begin{tabular}{lcccccccc}
\hline & $\begin{array}{c}\text { Kwon et al., } \\
2010^{27}\end{array}$ & $\begin{array}{c}\text { El-Sayed } \\
\text { et al., } 2012^{30}\end{array}$ & $\begin{array}{c}\text { Pereira et al., } \\
2013^{32}\end{array}$ & $\begin{array}{c}\text { Ha et al., } \\
2014^{33}\end{array}$ & $\begin{array}{c}\text { Pataka et al., } \\
2014^{\ddagger 34}\end{array}$ & $\begin{array}{c}\text { Kim et al., } \\
2015^{3}\end{array}$ & $\begin{array}{c}\text { Prasad et al., } \\
2017^{37}\end{array}$ & $\begin{array}{c}\text { Miller et al., } \\
2018^{38}\end{array}$ \\
\hline Berlin & & & & & & & & \\
SN (\%) & 72.0 & 95.5 & 91.0 & 75.0 & 78.0 & 75.5 & 87.5 & 92.3 \\
SP (\%) & 43.0 & 7.4 & 28.0 & 32.1 & 18.0 & 35.4 & 37.8 & 18.2 \\
PPV (\%) & 72.0 & 87.1 & 73.4 & 62.4 & 16.5 & 62.1 & 72.1 & 48.8 \\
NPV (\%) & 43.0 & 20.0 & 57.9 & 46.2 & 80.4 & 50.6 & 62.2 & 73.7 \\
LR(+)/LR(-) & $1.3^{*} / 0.7^{\dagger}$ & $1.0 / 0.6$ & $1.3 / 0.3$ & $1.1^{*} / 0.8^{\dagger}$ & $1.0 / 1.2$ & $1.2^{*} / 0.7^{\dagger}$ & $1.4 / 0.3$ & $1.1^{*} / 0.4^{\dagger}$ \\
STOP & & & & & & \\
SN (\%) & 97.0 & 94.4 & - & 76.2 & 92.7 & - & 91.9 & - \\
SP (\%) & 20.0 & 25.9 & - & 40.0 & 6.6 & - & 39.2 & - \\
PPV (\%) & 71.0 & 89.3 & - & 66.0 & 17.3 & - & 73.5 & - \\
NPV (\%) & 78.0 & 41.2 & - & 52.4 & 72.0 & - & 72.5 & - \\
LR(+)/LR(-) & $1.2^{*} / 0.2^{\dagger}$ & $1.3 / 0.2$ & - & $1.3^{*} / 0.6^{\dagger}$ & $1 / 1.1$ & - & $1.5 / 0.2$ & - \\
STOP-Bang & & & & & & & \\
SN (\%) & - & 97.7 & 93.0 & 85.7 & 94.8 & 98.0 & 93.4 & 95.4 \\
SP (\%) & - & 3.7 & 28.0 & 45.5 & 5.5 & 10.6 & 39.2 & 13.0 \\
PPV (\%) & - & 86.9 & 73.9 & 70.6 & 17.0 & 60.6 & 73.8 & 48.1 \\
NPV (\%) & - & 20.0 & 64.7 & 67.6 & 84.0 & 78.8 & 76.3 & 76.9 \\
LR(+)/LR(-) & - & $1.0 / 0.6$ & $1.3 / 0.2$ & $1.6^{*} / 0.3^{\dagger}$ & $1.0 / 0.9$ & $1.1^{*} / 0.2^{\dagger}$ & $1.5 / 0.2$ & $1.1^{*} / 0.4^{\dagger}$ \\
\hline
\end{tabular}

*the likelihood ratio for a positive result=SN/(1-SP), ${ }^{t}$ the likelihood ratio for a negative result=(1-SN)/SP, ${ }^{\ddagger}$ area under the receiver operating characteristic curve: Berlin, 0.48; STOP, 0.50; STOP-Bang, 0.52. SN: sensitivity, SP: specificity, PPV: positive predictive value, NPV: negative predictive value, $\mathrm{LR}(+)$ : positive likelihood ratio, $\mathrm{LR}(-)$ : negative likelihood ratio 
Table 6. Diagnostic accuracy of screening tools in a sleep clinical setting (Apnea-Hypopnea Index $\geq 30$ events/hr)

\begin{tabular}{|c|c|c|c|c|c|c|c|c|}
\hline & $\begin{array}{c}\text { Kwon et al., } \\
2010^{27}\end{array}$ & $\begin{array}{c}\text { El-Sayed } \\
\text { et al., } 2012^{30}\end{array}$ & $\begin{array}{c}\text { Pereira et al., } \\
2013^{32}\end{array}$ & $\begin{array}{c}\text { Ha et al., } \\
2014^{33}\end{array}$ & $\begin{array}{c}\text { Pataka et al., } \\
2014^{\ddagger^{34}}\end{array}$ & $\begin{array}{c}\text { Kim et al., } \\
2015^{3}\end{array}$ & $\begin{array}{c}\text { Prasad et al., } \\
2017^{37}\end{array}$ & $\begin{array}{c}\text { Miller et al., } \\
2018^{38}\end{array}$ \\
\hline \multicolumn{9}{|l|}{ Berlin } \\
\hline $\mathrm{SN}(\%)$ & 78.0 & 97.3 & 89.0 & 80.4 & 90.0 & - & 89.4 & 90.3 \\
\hline SP (\%) & 39.0 & 10.7 & 18.0 & 32.6 & 28.5 & - & 32.1 & 14.4 \\
\hline PPV (\%) & 43.0 & 74.2 & 45.9 & 40.6 & 56.0 & - & 56.4 & 22.8 \\
\hline NPV (\%) & 74.0 & 60.0 & 68.4 & 74.4 & 74.0 & - & 75.6 & 84.2 \\
\hline $\operatorname{LR}(+) / \operatorname{LR}(-)$ & $1.3^{*} / 0.6^{\dagger}$ & $1.1 / 0.3$ & $1.1 / 0.6$ & $1.2^{*} / 0.6^{\dagger}$ & $1.3 / 0.4$ & - & $1.3 / 0.3$ & $1.1^{*} / 0.7^{\dagger}$ \\
\hline \multicolumn{9}{|l|}{ STOP } \\
\hline $\mathrm{SN}(\%)$ & 98.0 & 96.0 & - & 80.4 & 97.0 & - & 95.2 & - \\
\hline SP (\%) & 12.0 & 19.6 & - & 36.4 & 11.0 & - & 33.0 & - \\
\hline PPV (\%) & 40.0 & 72.6 & - & 42.3 & 52.3 & - & 58.2 & - \\
\hline NPV (\%) & 89.0 & 64.7 & - & 76.2 & 78.4 & - & 87.5 & - \\
\hline $\operatorname{LR}(+) / \operatorname{LR}(-)$ & $1.1^{*} / 0.2^{\dagger}$ & $1.2 / 0.2$ & - & $1.3^{*} / 0.5^{\dagger}$ & $1.1 / 0.3$ & - & $1.4 / 0.2$ & - \\
\hline \multicolumn{9}{|l|}{ STOP-Bang } \\
\hline SN (\%) & - & 98.7 & 96.0 & 86.3 & 98.7 & - & 96.2 & 96.8 \\
\hline SP (\%) & - & 5.4 & 21.0 & 34.1 & 9.9 & - & 32.1 & 10.8 \\
\hline PPV (\%) & - & 73.4 & 48.6 & 43.1 & 52.7 & - & 58.1 & 23.3 \\
\hline NPV (\%) & - & 60.0 & 88.2 & 81.1 & 88.4 & - & 89.5 & 92.3 \\
\hline $\operatorname{LR}(+) / \operatorname{LR}(-)$ & - & $1.0 / 0.3$ & $1.2 / 0.2$ & $1.3^{*} / 0.4^{\dagger}$ & $1.1 / 0.1$ & - & $1.4 / 0.1$ & $1.1^{*} / 0.3^{\dagger}$ \\
\hline
\end{tabular}

*the likelihood ratio for a positive result=SN/(1-SP), ${ }^{\dagger}$ the likelihood ratio for a negative result=(1-SN)/SP, ${ }^{\ddagger}$ area under the receiver operating characteristic curve: Berlin, 0.60; STOP, 0.63; STOP-Bang, 0.72. SN: sensitivity, SP: specificity, PPV: positive predictive value, NPV: negative predictive value, $\mathrm{LR}(+)$ : positive likelihood ratio, $\mathrm{LR}(-)$ : negative likelihood ratio

0.52 로 나타나, 세 가지 설문 모두 OSA 진단에 정확성이 부 족한 도구라 여겨진다. ${ }^{44}$ 반면, STOP-Bang 설문은 $\mathrm{AHI}$ 절 단 값을 30 으로 적용하였을 때 $\mathrm{AUC}$ 수치가 0.72 로 나타나 중증 OSA 환자 선별에는 중등도의 정확성을 나타냈다. 하 지만 $\mathrm{AHI}$ 절단 값 30 을 적용한 $\mathrm{AUC}$ 수치는 경증 $(5 \leq \mathrm{AHI}$ $<15)$ 이나 중등도 환자 $(15 \leq \mathrm{AHI}<30)$ 의 특성은 배제하는 결과를 초래하므로, 질병의 중증도를 고려하여 절단 값과 선 별도구를 결정해야 할 것이다.

최근 아이슬란드 인구를 대상으로 OSA 유병률과 수면무 호흡 관련 증상을 조사한 연구에 따르면, 40 65세 성인의 약 20\%가 중등도에서 중증의 OSA를 가지고 있으나, 이들 대부분이 수면무호흡과 관련된 주관적 증상 즉, 주간 졸음, 각성 시 구강 건조, 아침 두통, 수면 시작의 어려움 등을 거 의 느끼지 못하는 것으로 나타났다. ${ }^{45}$ 이는 전체 인구 중 무 증상(asymptomatic) OSA를 가진 환자가 적지 않고, 더불어 무증상으로 인해 이들이 진단과 치료를 목적으로 수면클리 닉에 내원할 가능성이 낮음을 의미한다. 또한, $\mathrm{Ye}$ 등에 따르 면 수면클리닉의 OSA 환자는 '수면장애군(disturbed sleep group)', '최소 증상군(minimally symptomatic group)', '과도 한 주간졸음군(excessive daytime sleepiness group)'과 같은
세 가지 임상 군집(clinical clusters)으로 구분이 가능하고, ${ }^{46}$ Keenan 등이 이 연구를 아시아를 포함한 여러 국가로 조사 대상을 확대하였을 때도 유사한 결과가 확인되었다. ${ }^{47}$ 이는 일반적인 지역사회 인구 기반 연구와 증상 발현으로 수면클 리닉을 내원한 환자 대상 연구는 연구 간 참여 대상자의 특 징이 본질적으로 다를 수밖에 없음을 시사한다. 따라서 OSA 의 유병률이나 실태 조사 시에는 해부학적 및 생리학적 요 인, 수면무호흡과 관련된 특징적 증상이나 일상생활 증상 등 과 같은 수면무호흡의 위험인자들이 모두 반영된 선별도구 를 사용할 것을 제안한다. 그리고 수면클리닉 내원 환자를 대상으로 연구하거나 진단을 내릴 때는 BMI와 목둘레 같은 신체 계측치와 수면무호흡 관련 증상을 반영한 선별도구를 사용하는 것이 유용할 것으로 여겨진다.

\section{결 론}

OSA 진단을 위한 환자의 경제적 부담을 덜어주고자 정부 가 PSG 항목에 건강보험을 적용한 후 수면장애의 진단, 치 료 및 관리에 대한 국민적 관심이 증가하고 있다. 건강보험 이 적용된다 하더라도 $\mathrm{PSG}$ 를 위한 비용이나 시간 소요가 
많으므로, 비용 효과적이면서 임상적 의사결정에 도움을 줄 수 있는 선별도구의 활용이 필요해 보인다.

본 종설을 통해 지역사회 인구 기반 연구 시 중증 OSA를 선별해내는 데 Berlin 설문이 가장 유용한 도구이나 여성 노 인 환자를 예측함에 있어서는 민감도와 양성예측도가 낮았 고 $\mathrm{AHI}$ 절단 값이 높아질수록 민감도는 높아지나 특이도는 낮아지는 것을 알 수 있었다. OSA와 관련된 증상 발현으로 수면클리닉을 내원한 환자에게 Berlin 설문을 적용하였을 때 민감도, 특이도, 양성/음성 예측도, 양성/음성 우도비는 연구 간 큰 차이를 보였고, AUC는 0.5점대에 머물러 Berlin 설문의 진단적 유용성은 그리 높지 않은 것으로 확인되었다. 또한 Berlin, STOP, STOP-Bang 설문의 OSA 진단적 유용 성을 수면클리닉 내원 환자에서 비교하였을 때, 민감도는 국 내 환자에서 STOP-Bang이 가장 높고 특이도는 Berlin이 가장 높은 것으로 파악되었다.

따라서 Berlin 설문을 임상에서 적용할 때는 환자의 성별, 연령, 비만도, 동반 질환 등의 특성과 $\mathrm{AHI}$ 절단 값 등이 고 려되어야 하고, 연구 수행 시에는 연구 대상자의 수나 모집 단의 특성을 함께 고려해야 할 것으로 사료된다. 또한 임상 에서 STOP과 STOP-Bang 설문을 적용할 때 설문에 포함된 $\mathrm{OSA}$ 의 비특이적 증상(피곤함, 고혈압, 50세 이상의 연령, 남 성)으로 OSA 양성 판정을 받은 대상자에 대해서는 신체 계 측 및 생리적 지수에 대한 추가 검사를 통해 설문의 특이도 를 높이는 전략이 필요하다 여겨진다. 그러나 OSA 진단을 위한 선별도구의 적용 결과는 진단적 유용성의 주요 지표를 무엇으로 삼을 것인가에 의해서도 달라질 수 있으므로, 이러 한 점들에 주의하면서 선별도구를 선택하는 데 신중을 기해 야 할 것이다.

\section{Conflicts of Interest}

The authors have no potential conflicts of interest to disclose.

\section{ORCID iDs}

Hyunju Yang

Hyunyoung Park

https://orcid.org/0000-0003-0027-2834

https://orcid.org/0000-0003-3668-7900

\section{Author Contributions}

Conceptualization: Hyunju Yang, Hyunyoung Park. Data curation: Hyunju Yang. Formal analysis: Hyunju Yang. Investigation: Hyunju Yang, Hyunyoung Park. Methodology: Hyunju Yang, Hyunyoung Park. Validation: Hyunju Yang, Hyunyoung Park. Visualization: Hyunju Yang, Hyunyoung Park. Writing_original draft: Hyunju Yang. Writing_review \& editing: Hyunyoung Park.

\section{REFERENCES}

1. Berry RB, Budhiraja R, Gottlieb DJ, et al. Rules for scoring respiratory events in sleep: update of the 2007 AASM manual for the scoring of sleep and associated events. Deliberations of the sleep apnea definitions task force of the American Academy of Sleep Medicine. J Clin
Sleep Med 2012;8:597-619.

2. Young T, Palta M, Dempsey J, Skatrud J, Weber S, Badr S. The occurrence of sleep-disordered breathing among middle-aged adults. $N$ Engl J Med 1993;328:1230-1235.

3. Kim B, Lee EM, Chung YS, Kim WS, Lee SA. The utility of three screening questionnaires for obstructive sleep apnea in a sleep clinic setting. Yonsei Med J 2015;56:684-690.

4. Netzer NC, Hoegel JJ, Loube D, et al.; Sleep in Primary Care International Study Group. Prevalence of symptoms and risk of sleep apnea in primary care. Chest 2003;124:1406-1414.

5. McNicholas WT, Rodenstein D. Sleep apnoea and driving risk: the need for regulation. Eur Respir Rev 2015;24:602-606.

6. Punjabi NM. The epidemiology of adult obstructive sleep apnea. Proc Am Thorac Soc 2008;5:136-143.

7. Somers VK, White DP, Amin R, et al. Sleep apnea and cardiovascular disease: an American Heart Association/American College of Cardiology Foundation Scientific Statement from the American Heart Association Council for High Blood Pressure Research Professional Education Committee, Council on Clinical Cardiology, Stroke Council, and Council on Cardiovascular Nursing. J Am Coll Cardiol 2008;52:686-717.

8. Gami AS, Howard DE, Olson EJ, Somers VK. Day-night pattern of sudden death in obstructive sleep apnea. N Engl J Med 2005;352:1206-1214.

9. Marin JM, Carrizo SJ, Vicente E, Agusti AG. Long-term cardiovascular outcomes in men with obstructive sleep apnoea-hypopnoea with or without treatment with continuous positive airway pressure: an observational study. Lancet 2005;365:1046-1053.

10. Kaditis AG, Alonso Alvarez ML, Boudewyns A, et al. Obstructive sleep disordered breathing in 2- to 18-year-old children: diagnosis and management. Eur Respir J 2016;47:69-94.

11. Littner M. Polysomnography in the diagnosis of the obstructive sleep apnea-hypopnea syndrome: where do we draw the line? Chest 2000;11 8:286-288.

12. Ministry of Health and Welfare. Health insurance application to polysomnography and continuous positive airway pressure treatment. Ministry of Health and Welfare 2018 [cited 2018 Mar 20; updated 2018 Mar 21]. URL:http://www.mohw.go.kr/react/al/sal0301vw.jsp? PAR_MENU_ID=04\&MENU_ID=0403\&CONT_SEQ=344261\& page $=1$. Access 2019 Feb 20.

13. Netzer NC, Stoohs RA, Netzer CM, Clark K, Strohl KP. Using the Berlin questionnaire to identify patients at risk for the sleep apnea syndrome. Ann Intern Med 1999;131:485-491.

14. Ahmadi N, Chung SA, Gibbs A, Shapiro CM. The Berlin questionnaire for sleep apnea in a sleep clinic population: relationship to polysomnographic measurement of respiratory disturbance. Sleep Breath 2008;12:39-45.

15. Chiu HY, Chen PY, Chuang LP, et al. Diagnostic accuracy of the Berlin questionnaire, STOP-BANG, STOP, and Epworth sleepiness scale in detecting obstructive sleep apnea: a bivariate meta-analysis. Sleep Med Rev 2017;36:57-70.

16. Amra B, Rahmati B, Soltaninejad F, Feizi A. Screening auestionnaires for obstructive sleep apnea: an updated systematic review. Oman Med J 2018;33:184-192.

17. Chung F, Abdullah HR, Liao P. STOP-Bang questionnaire: a practical approach to screen for obstructive sleep apnea. Chest 2016;149:631-638.

18. Sforza E, Chouchou F, Pichot V, Herrmann F, Barthélémy JC, Roche F. Is the Berlin questionnaire a useful tool to diagnose obstructive sleep apnea in the elderly? Sleep Med 2011;12:142-146.

19. Kang K, Park KS, Kim JE, et al. Usefulness of the Berlin questionnaire to identify patients at high risk for obstructive sleep apnea: a population-based door-to-door study. Sleep Breath 2013;17:803-810.

20. Tan A, Yin JD, Tan LW, van Dam RM, Cheung YY, Lee CH. Using the Berlin questionnaire to predict obstructive sleep apnea in the general population. J Clin Sleep Med 2017;13:427-432.

21. Tan A, Yin JD, Tan LW, van Dam RM, Cheung YY, Lee CH. Predicting obstructive sleep apnea using the STOP-Bang questionnaire in the 
general population. Sleep Med 2016;27-28:66-71.

22. Silva GE, Vana KD, Goodwin JL, Sherrill DL, Quan SF. Identification of patients with sleep disordered breathing: comparing the four-variable screening tool, STOP, STOP-Bang, and Epworth sleepiness scales. J Clin Sleep Med 2011;7:467-472.

23. Kapsimalis F, Kryger M. Sleep breathing disorders in the U.S. female population. J Womens Health (Larchmt) 2009;18:1211-1219.

24. Roth T. Insomnia: definition, prevalence, etiology, and consequences. $J$ Clin Sleep Med 2007;3(5 Suppl):S7-S10.

25. Ministry of Health and Welfare. Standards and methods of insurance reimbursement for medical expenses. Ministry of Health and Welfare 2018 [updated 2018 Dec 31]. URL:http://www.mohw.go.kr/react/jb/ sjb0406vw.jsp?PAR_MENU_ID $=03 \&$ MENU_ID $=030406 \&$ page $=1$ \&CONT_SEQ=347234. Accessed 2019 Feb 20.

26. Collop NA, Anderson WM, Boehlecke B, et al. Clinical guidelines for the use of unattended portable monitors in the diagnosis of obstructive sleep apnea in adult patients. Portable Monitoring Task Force of the American Academy of Sleep Medicine. J Clin Sleep Med 2007;3: 737-747.

27. Kwon C, Shin SY, Lee KH, Cho JS, Kim SW. Usefulness of Berlin and STOP questionnaires as a screening test for sleep apnea in Korea. Korean J Otorhinolaryngol-Head Neck Surg 2010;53:768-772.

28. Sagaspe P, Leger D, Taillard J, Bayon V, Chaumet G, Philip P. Might the Berlin Sleep Questionnaire applied to bed partners be used to screen sleep apneic patients? Sleep Med 2010;11:479-483.

29. Kang HH, Kang JY, Lee SH, Moon HS. The usefulness of the Berlin questionnaire as a screening for obstructive sleep apnea in a sleep clinic population. Sleep Med Psychophysiol 2011;18:82-86.

30. El-Sayed IH. Comparison of four sleep questionnaires for screening obstructive sleep apnea. Egypt J Chest Dis Tuberc 2012;61:433-441.

31. Amra B, Nouranian E, Golshan M, Fietze I, Penzel T. Validation of the persian version of Berlin sleep questionnaire for diagnosing obstructive sleep apnea. Int J Prev Med 2013;4:334-339.

32. Pereira EJ, Driver HS, Stewart SC, Fitzpatrick MF. Comparing a combination of validated questionnaires and level III portable monitor with polysomnography to diagnose and exclude sleep apnea. J Clin Sleep Med 2013;9:1259-1266.

33. Ha SC, Lee DL, Abdullah VJ, van Hasselt CA. Evaluation and validation of four translated Chinese questionnaires for obstructive sleep apnea patients in Hong Kong. Sleep Breath 2014;18:715-721.

34. Pataka A, Daskalopoulou E, Kalamaras G, Fekete Passa K, Argyropou- lou P. Evaluation of five different questionnaires for assessing sleep apnea syndrome in a sleep clinic. Sleep Med 2014;15:776-781.

35. Ulasli SS, Gunay E, Koyuncu T, et al. Predictive value of Berlin questionnaire and Epworth sleepiness scale for obstructive sleep apnea in a sleep clinic population. Clin Respir J 2014;8:292-296.

36. Yüceege M, Fırat H, Sever Ö, Demir A, Ardıç S. The effect of adding gender item to Berlin questionnaire in determining obstructive sleep apnea in sleep clinics. Ann Thorac Med 2015;10:25-28.

37. Prasad KT, Sehgal IS, Agarwal R, Nath Aggarwal A, Behera D, Dhooria S. Assessing the likelihood of obstructive sleep apnea: a comparison of nine screening questionnaires. Sleep Breath 2017;21:909-917.

38. Miller JN, Kupzyk KA, Zimmerman L, et al. Comparisons of measures used to screen for obstructive sleep apnea in patients referred to a sleep clinic. Sleep Med 2018;51:15-21.

39. Peppard PE, Young T, Palta M, Dempsey J, Skatrud J. Longitudinal study of moderate weight change and sleep-disordered breathing. JAMA 2000;284:3015-3021.

40. Resta O, Foschino-Barbaro MP, Legari G, et al. Sleep-related breathing disorders, loud snoring and excessive daytime sleepiness in obese subjects. Int J Obes Relat Metab Disord 2001;25:669-675.

41. Kapur VK, Auckley DH, Chowdhuri S, et al. Clinical practice guideline for diagnostic testing for adult obstructive sleep apnea: an American Academy of Sleep Medicine clinical practice guideline. J Clin Sleep Med 2017;13:479-504.

42. Epstein LJ, Kristo D, Strollo PJ Jr, et al.; Adult Obstructive Sleep Apnea Task Force of the American Academy of Sleep Medicine. Clinical guideline for the evaluation, management and long-term care of obstructive sleep apnea in adults. J Clin Sleep Med 2009;5:263-276.

43. Barraclough PJ. Common method variance and other sources of bias in road traffic research [dissertation]. Brisbane: Queensland University of Technology;2017.

44. Song SW. Assess the accuracy of diagnostic tools. Korean J Fam Pract 2018;8:1-2.

45. Arnardottir ES, Bjornsdottir E, Olafsdottir KA, Benediktsdottir B, Gislason T. Obstructive sleep apnoea in the general population: highly prevalent but minimal symptoms. Eur Respir J 2016;47:194-202.

46. Ye L, Pien GW, Ratcliffe SJ, et al. The different clinical faces of obstructive sleep apnoea: a cluster analysis. Eur Respir J 2014;44:1600-1607.

47. Keenan BT, Kim J, Singh B, et al. Recognizable clinical subtypes of obstructive sleep apnea across international sleep centers: a cluster analysis. Sleep 2018;41:1-14. 